\section{Endocarditis infecciosa por Streptococcus pluranimalium: reporte de un caso}

\section{Infective endocarditis by Streptococcus pluranimalium: Case report}

La endocarditis infecciosa es una enfermedad cardiaca grave, con pocos cambios en la morbimortalidad en los últimos 30 años, a pesar de las mejoras en el diagnóstico y los procedimientos terapéuticos. En la actualidad, hasta el $31 \%$ de las endocarditis son con cultivos negativos, pero en aquellos pacientes con cultivos positivos, se encuentran como agentes etiológicos más frecuentes las especies de estafilococos, estreptococos y enterococo ${ }^{1}$. Sin embargo, el Streptococcus pluranimalium (S. pluranimalium) es un patógeno que no hace parte de los gérmenes típicos de esta infección cardiaca, y de hecho parece ser un patógeno nuevo en humanos, ya que en la revisión de la literatura, se encuentran reportes de procesos infecciosos en animales, y escasos reportes de casos de infecciones en humanos. Se reporta, entonces, el primer caso conocido de endocarditis infecciosa por S. pluranimalium en humanos en nuestro país, y el segundo a nivel mundial.

Describimos el caso de un paciente de sexo masculino, 25 años de edad, que ingresó a urgencias de nuestro hospital. El paciente es residente de área rural en contacto con diversos animales de corral, con antecedentes de síndrome de Down, retraso mental grave e insuficiencia renal crónica (atrofia renal izquierda). Consultó por fiebre intermitente de 3 meses de evolución, pérdida de peso y diaforesis nocturna. En la última semana, se tornó disneico, motivo por el cual sus familiares deciden consultar.

Hallazgos al examen físico: presión arterial 120/ $80 \mathrm{mmHg}$, frecuencia cardíaca $100 \mathrm{lpm}$, frecuencia respiratoria $22 \mathrm{~min}$ y saturación de oxígeno: $88 \%$. Dentadura en buen estado, sin distensión yugular, ni adenopatías cervicales. Soplo cardiaco sistólico grado $2 / 4$ en foco mitral, además de crépitos en ambas bases pulmonares. Abdomen sin alteraciones. Edema en miembros inferiores, sin lesiones en piel ni focalización neurológica.

La impresión diagnóstica inicial fue sepsis de origen pulmonar. Se inició tratamiento con piperacilina tazobactam $4.5 \mathrm{~g}$ iv cada $8 \mathrm{~h}$ (previa toma de hemocultivos), durante 4 días.

Los paraclínicos documentaron: anemia normocítica normocrómica (hemoglobina: $9 \mathrm{~g} / \mathrm{dl}$ ) asociada a trombocitopenia $\left(100,000 / \mathrm{mm}^{3}\right)$, hipoalbuminemia $(3 \mathrm{~g} / \mathrm{dl})$, hematuria microscópica, proteinuria y fallo renal (creatinina: $1.6 \mathrm{mg} / \mathrm{dl}$ ), por lo que se configuró una glomerulonefritis; además, la radiografía de tórax y posteriormente una tomografía de tórax descartaron no solo infección pulmonar, sino linfadenopatías y hepatoesplenomegalia, aunque mostraron signos de congestión pulmonar. Posteriormente se le practica al paciente, una ecocardiografía transesofágica que evidenció: prolapso de valva anterior de la válvula mitral, con insuficiencia severa e imagen de $2.1 \times 1.1 \mathrm{~cm}$ en el tercio distal, compatible con vegetación endocárdica (fig. 1).

Los hemocultivos tomados al ingreso reportaron crecimiento en las 2 botellas (19 y $20 \mathrm{~h}$ de incubación) de cocos Gram positivos catalasa negativos, de los que se realizó

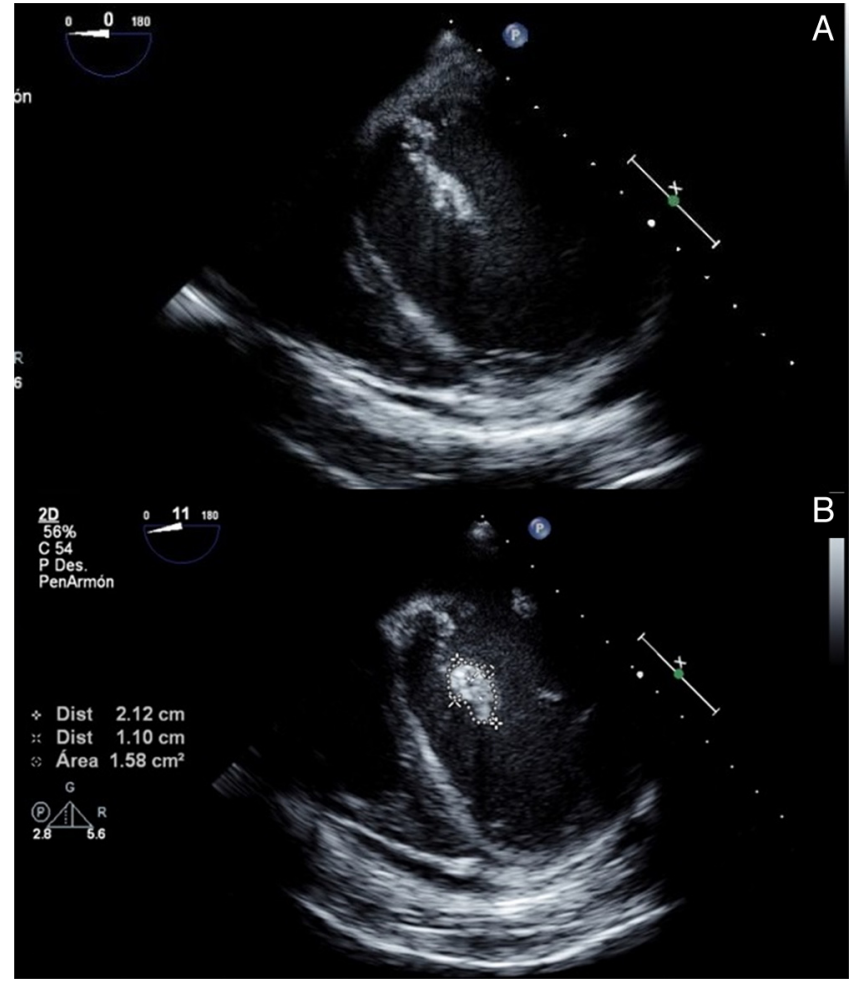

Figura 1 Ecocardiografía transesofágica a 0 grados: A) Se evidencia una vegetación en la valva anterior de la válvula mitral. B) Se muestran los diámetros y el área de la vegetación.

identificación fenotípica de especie en equipo automatizado VITEK2 ${ }^{\circledR}$ (bioMérieux) como S. pluranimalium, con sensibilidad in vitro a penicilina, clindamicina, eritromicina, linezolid, cefotaxima y vancomicina por el método de KirbyBauer. Basados en los criterios de Duke modificados ${ }^{1}$, se hace el diagnóstico de endocarditis infecciosa, cumpliendo un criterio mayor (presencia de vegetación en la ecocardiografía) y 4 menores (condición cardiaca predisponente, fiebre, glomerulonefritis y hemocultivos positivos de un germen que no cumplía las características del criterio mayor).

Debido a que el paciente tenía insuficiencia mitral severa con signos y síntomas de fallo cardiaco, y el tamaño de la vegetación mayor de $15 \mathrm{~mm}$, se consideró trasladarlo a cirugía para cambio valvular mitral (válvula biológica). Es de anotar, que a pesar de ser un paciente joven, se definió implante de válvula biológica, debido a que las condiciones sociales del paciente (retardo mental grave y vivir en zona rural), le hacían muy difícil los desplazamientos para asistir a controles de anticoagulación. Se realizaron hemocultivos de control 8 días después del inicio de la antibioticoterapia sin crecimiento microbiológico, y terminó tratamiento antibiótico con ampicilina sulbactam ajustado según el perfil de sensibilidad hasta completar 28 días, de acuerdo a la recomendación de las guías para el tratamiento de estreptococos ${ }^{1}$. No recibió gentamicina por su compromiso renal. El paciente fue dado de alta sin complicaciones o secuelas.

Luego de su descripción inicial por Devriese et al. en 1999 , el S. pluranimalium se aisló inicialmente en mastitis bobina, vagina, cérvix y amígdalas de bovinos, y lesiones en pulmón de canarios $^{2}$, con descripciones posteriores 
de infección en un gato, cabras $^{3}$, y de meningoventriculitis en terneros neonatos con parto pretérmino y endocarditis en pollos adultos de engorde ${ }^{3,4}$.

Se realizó una amplia búsqueda en la literatura en diferentes bases de datos médicas y a través de búsquedas libres, encontrando que recientemente se han realizado publicaciones de infecciones en humanos por S. pluranimalium. Dichos reportes incluyen el realizado por Paolucci et al. ${ }^{5}$, quienes en un estudio de infecciones en pacientes neutropénicos (usando para los cultivos el sistema de identificación bacteriana VITEK $2^{\circledR}$ ), reportan que se tuvo un aislamiento de $S$. pluranimalium en un paciente, pero no ofrecen datos clínicos adicionales. El segundo reporte es el de Jacob et al. ${ }^{6}$, quienes describieron el caso de una mujer de 53 años que presentó artritis séptica de su rodilla derecha, con posterior choque séptico y disfunción multiorgánica que le causó la muerte, y a quien se le aisló S. pluranimalium en los cultivos, también identificados con el sistema VITEK2 ${ }^{\circledR}$. Por otro lado, Aryasinghe et al. ${ }^{3}$, describieron un caso de empiema subdural en un varón de 17 años de raza negra, con crecimiento de $S$. pluranimalium identificado con el sistema VITEK $2^{\circledR}$, logrando recuperación del paciente con el drenaje quirúrgico del empiema subdural y terapia antibiótica con meropenem $2 \mathrm{~g}$ cada $8 \mathrm{~h}$ y vancomicina $1 \mathrm{~g}$ cada $12 \mathrm{~h}$ en forma iv, durante 6 semanas. Finalmente, Fotoglidis et al. ${ }^{7}$, publicaron un caso de endocarditis infecciosa en un paciente de 37 años de edad, adicto a drogas iv, a quien se le aisló $S$. pluranimalium con el sistema automatizado VITEK $2^{\circledR}$, con un desenlace fatal, a pesar de la terapia antibiótica y el manejo quirúrgico. Se deben tener presentes las dificultades para la identificación de especies de este grupo de estreptococos con los equipos actuales de identificación microbiológica como es el VITEK $2^{\circledR}$, lo que puede ser un desafío para los laboratorios de microbiología clínica.

En la búsqueda de la literatura realizada, no encontramos casos reportados de endocarditis infecciosa en humanos por el germen, en mención diferente al de Fotoglidis et al. ${ }^{7}$, por lo que, este sería el primer caso de endocarditis infecciosa en humanos por S. pluranimalium en nuestro país y el segundo reporte en el mundo, aunque con la diferencia, de que en el caso que reportamos se obtuvo un resultado exitoso, ya que se logró la curación del proceso infeccioso con la terapia previamente descrita.

\section{Financiación}

No se recibió patrocinio de ningún tipo para llevar a cabo este artículo.

\section{Conflicto de intereses}

Los autores declaran no tener ningún conflicto de intereses.

\section{Agradecimientos}

Agradecemos al paciente y sus familiares, y al Hospital Universitario San Vicente Fundación, que facilitó la información de la historia clínica y ayudas diagnósticas.

\section{Bibliografía}

1. Habib G, Lancelloti P, Antunes MJ, et al. 2015 ESC Guidelines for the management of infective endocarditis: The Task Force for the management of Infective Endocarditis of the European Society of Cardiology (ESC). Eur Heart J. 2015;36:3075-123.

2. Facklam R. What happened to the streptococci: Overview of taxonomic and nomenclature changes. Clin Microbiol Rev. 2002;15:613-30.

3. Hedegaard L, Christensen H, Chadfield MS, et al. Association of Streptococcus pluranimalium with valvular endocarditis and septicaemia in adult broiler parents. Avian Pathol. 2009;38: 155-60.

4. Aryasinghe L, Sabbar S, Kazim Y, et al. Streptococcus pluranimalium: A novel human pathogen? Int J Surg Case Rep. 2015;5:1242-6.

5. Paolucci M, Stanzani M, Melchionda F, et al. Routine use of a real-time polymerase chain reaction method for detection of bloodstream infections in neutropaenic patients. Diagn Microbiol Infect Dis. 2013;75:130-4.

6. Jacob E, Kiran S, Jithendranath A, et al. Streptococcus Pluranimalium-Close Encounter of A New Kind Internet. [consultado 3 Ene 2015]. Disponible en: http:/ / www.japi.org/oral_Feb_ 2014/poster_Infectious_Diseases.html

7. Fotoglidis A, Pagourelias E, Kyriakou P, et al. Endocarditis caused by unusual Streptococcus species (Streptococcus pluranimalium). Hippokratia. 2015;19:182-5.

Edison Muñoz Ortiz ${ }^{a, *}$, Julián H. Ramírez Urrea ${ }^{\mathrm{b}}$, Santiago Atehortúa Muñoz ${ }^{\mathrm{c}}$ y

Edwin Fernando Arévalo Guerrero ${ }^{a}$

a Servicio de Cardiología, Hospital Universitario San

Vicente Fundación y Universidad de Antioquia, Medellín, Colombia

b Servicio de Medicina Interna, Hospital Universitario San

Vicente Fundación y Universidad de Antioquia, Medellín, Colombia

c Servicio de Laboratorio, Hospital Universitario San

Vicente Fundación, Medellín, Colombia

* Autor para correspondencia. Servicio de Cardiología, Hospital Universitario San Vicente Fundación, Calle 64 \#51D-154, bloque 3, piso 2, Medellín, Colombia,

Teléfono: 57-4-2192420.

Correo electrónico: edisonhoy@yahoo.es (E. Muñoz Ortiz).

http://dx.doi.org/10.1016/j.acmx.2016.04.004 